\title{
Photoacoustic imaging of kidney fibrosis for assessing pretransplant organ quality
}

\author{
Eno Hysi, ${ }^{1,2}$ Xiaolin He, ${ }^{2,3,4}$ Muhannad N. Fadhel, ${ }^{1,2}$ Tianzhou Zhang, ${ }^{3,4}$ Adriana Krizova, ${ }^{4,5}$ \\ Michael Ordon,, ${ }^{4,5,6}$ Monica Farcas, ${ }^{4,5,6}$ Kenneth T. Pace, ${ }^{4,5,6}$ Victoria Mintsopoulos, ${ }^{4,7}$ Warren L. Lee, ${ }^{4,7}$ \\ Michael C. Kolios, ${ }^{1,2}$ and Darren A. Yuen ${ }^{2,3,4}$ \\ 'Department of Physics, Ryerson University, Toronto, Canada. ${ }^{2}$ Institute for Biomedical Engineering, Science and \\ Technology (iBEST), a partnership between Ryerson University and St. Michael's Hospital, Toronto, Canada. ${ }^{3}$ Division of \\ Nephrology, Department of Medicine, St. Michael's Hospital, Unity Health Toronto and University of Toronto, Toronto, \\ Canada. ${ }^{4}$ Keenan Research Centre for Biomedical Science and ${ }^{5}$ Department of Laboratory Medicine, St. Michael's Hospital, \\ Unity Health Toronto, Toronto, Canada. ${ }^{6}$ Division of Urology, Department of Surgery, St. Michael's Hospital, Unity Health \\ Toronto and University of Toronto, Toronto, Ontario, Canada. IInterdepartmental Division of Critical Care Medicine, St. \\ Michael's Hospital, University of Toronto, Toronto, Canada.
}

Roughly $10 \%$ of the world's population has chronic kidney disease (CKD). In its advanced stages, CKD greatly increases the risk of hospitalization and death. Although kidney transplantation has revolutionized the care of advanced CKD, clinicians have limited ways of assessing donor kidney quality. Thus, optimal donor kidney-recipient matching cannot be performed, meaning that some patients receive damaged kidneys that function poorly. Fibrosis is a form of chronic damage often present in donor kidneys, and it is an important predictor of future renal function. Currently, no safe, easy-to-perform technique exists that accurately quantifies renal fibrosis. We describe a potentially novel photoacoustic (PA) imaging technique that directly images collagen, the principal component of fibrotic tissue. PA imaging noninvasively quantifies whole kidney fibrotic burden in mice, and cortical fibrosis in pig and human kidneys, with outstanding accuracy and speed. Remarkably, 3-dimensional PA imaging exhibited sufficiently high resolution to capture intrarenal variations in collagen content. We further show that PA imaging can be performed in a setting that mimics human kidney transplantation, suggesting the potential for rapid clinical translation. Taken together, our data suggest that PA collagen imaging is a major advance in fibrosis quantification that could have widespread preclinical and clinical impact.

Authorship note: MCK and DAY contributed equally to this work.

Conflict of interest: The authors have declared that no conflict of interest exists.

Copyright: (c) 2020, American Society for Clinical Investigation.

Submitted: February 10, 2020

Accepted: April 9, 2020

Published: May 21, 2020

Reference information: JCI Insight. 2020;5(10):e136995.

https://doi.org/10.1172/jci.

insight.136995.

\section{Introduction}

Chronic kidney disease (CKD) is a major cause of morbidity and mortality. Despite decades of research, clinicians still have limited tools at their disposal to prevent disease progression. As such, many patients will progress to kidney failure, a condition that is associated with reduced quality of life, frequent and prolonged hospitalizations, and an increased risk of death $(1,2)$. Kidney transplantation can restore renal function and, thus, reduce mortality, improve quality of life, and reduce health care costs (3-5). However, a dramatic shortage of donor kidneys has meant that patients are often waiting years to receive a kidney $(1$, $2,6)$. With such a long wait, many patients become too ill to be transplanted or will even die on the wait list (7). To remedy this, kidneys from sicker donors are now frequently used, despite the fact that some of these kidneys are already damaged and will have a shorter life span $(8,9)$.

Fibrosis is a driver of CKD progression, being a final common pathway of injury activated not only in damaged donor kidneys, but in nearly all forms of $\operatorname{CKD}(10,11)$. Fibrosis of the renal cortex (the outer part of the kidney) is of particular importance, as scarring in this area of the kidney obliterates the glomerular capillaries responsible for blood filtration and, thus, impairs one of the principal functions of the kidney. Moreover, because the kidney is one of the few tissues in the body with 2 capillary beds connected in series, fibrotic glomerular capillary loss impairs the delivery of oxygen and nutrients to downstream peritubular capillaries, which supply the tubular epithelium of the cortex and the medulla (the inner part of the kidney) (12). Thus, fibrosis of the cortex not only reduces kidney function, but it also results in ischemic injury in other areas of the kidney, ultimately causing further renal damage (13). 
Not surprisingly, fibrosis is a powerful predictor of long-term kidney outcomes in transplantation and virtually all kidney diseases $(10,11)$, and it is an important disease process being actively targeted by experimental therapies (14).

Currently, the only available method for assessing renal fibrosis is histologic analysis, which requires invasive tissue sampling. In preclinical rodent models, this analysis can only be accomplished after sacrifice, whereas in humans, a needle biopsy of the kidney is required. Unfortunately, biopsies are limited by their small sampling size ( $<1 \%$ of 1 kidney) and by significant bleeding risks induced by the biopsy procedure ( 15 , 16). In the setting of kidney transplantation, these limitations mean that a biopsy is either not performed or, even if it is, quantification of fibrosis may not be reflective of overall renal scar burden (17). Thus, clinicians must currently rely on noninvasive surrogate measures of chronic kidney damage that, while somewhat informative, are limited in utility. As such, physicians must ultimately make decisions regarding the acceptance of a kidney and matching to a recipient based on incomplete and possibly incorrect information.

Imaging techniques have revolutionized our ability to noninvasively examine tissues. Unfortunately, no standard technology (conventional ultrasound, CT, or MRI) can reliably quantify renal fibrosis (18). The use of fibrosis-targeted contrast agents to augment standard imaging technologies has recently been described, but currently, their use has been limited by the toxicity and pharmacokinetics of these agents (19). Recently, however, the combination of ultrasound ("acoustic") with laser technology ("photo") has enabled contrast-free imaging of specific tissue components by taking advantage of their unique light absorption properties (20-22). Photoacoustics (PA) relies on the generation of acoustic signals from the thermoelastic expansion of tissues in response to noninvasive laser irradiation with light of particular wavelengths. Endogenous molecules such as melanin, hemoglobin, bilirubin, and lipids have unique light-absorption spectra, enabling high-resolution imaging of their spatial distribution by tuning the wavelength of laser illumination (23-25).

Here, we describe the first report to our knowledge of PA imaging of collagen in kidney tissue. Using ex vivo collagen phantom gels, as well as kidneys from mice, pigs, and humans, we show that PA collagen imaging demonstrates an ability to quantify both overall fibrotic burden and intrarenal variations in collagen content, when compared with gold standard invasive measures. Importantly, we show that PA collagen imaging can be easily and accurately performed on ex vivo human kidneys in a setting that mimics intraoperative kidney transplantation, illustrating the clinical translational potential of this imaging modality. To our knowledge, this is the first demonstration of a rapid, easy-to-perform, high-resolution, noninvasive imaging technique that can accurately quantify renal fibrotic burden.

\section{Results}

Development of a spectral unmixing algorithm to quantify collagen content using PA imaging. Kidneys, like all biological tissues, are composed of a complex mixture of molecules. Each molecule has its own light absorption properties and, thus, can act as a chromophore when illuminated by light of specific wavelengths (26) (Supplemental Figure 1; supplemental material available online with this article; https://doi.org/10.1172/ jci.insight.136995DS1). Light absorption results in thermoelastic expansion, such that a brief illumination pulse can lead to an expansion-contraction sequence that generates an acoustic wave that can be detected by an ultrasound transducer (Figure 1A). Thus, if a molecule is present in sufficient concentration, this pattern of light absorption can be used as a "molecular fingerprint" to estimate its amount and distribution within tissue using PA imaging (20).

Given the prevalence of type I collagen in kidney tissue and its overproduction in fibrotic kidneys (27), we decided to explore whether its concentration could be quantified using PA imaging. To develop and validate our technique, we first performed PA imaging of collagen phantom gels composed of porcine skin-derived gelatin (the major constituent of which is type I collagen). As blood is also a major component of renal tissue, and hemoglobin is a strong PA chromophore (28), we also mixed in varying amounts of porcine blood into the collagen phantoms. We scanned the blood-collagen phantom gels using a commercial PA system, illuminating the samples with light between 680 and $930 \mathrm{~nm}$. Using the generated PA data, we developed a spectral unmixing algorithm to identify the optimal wavelengths $(680,725$, and $755 \mathrm{~nm})$ that could best distinguish PA signals derived from excitation of hemoglobin versus that of collagen (Supplemental Methods and Figure 1B). Applying this unmixing algorithm to the scanned gels, we found that our PA imaging technique demonstrated outstanding accuracy in quantifying both collagen and hemoglobin content across a wide range of values (Figure 2). 
A

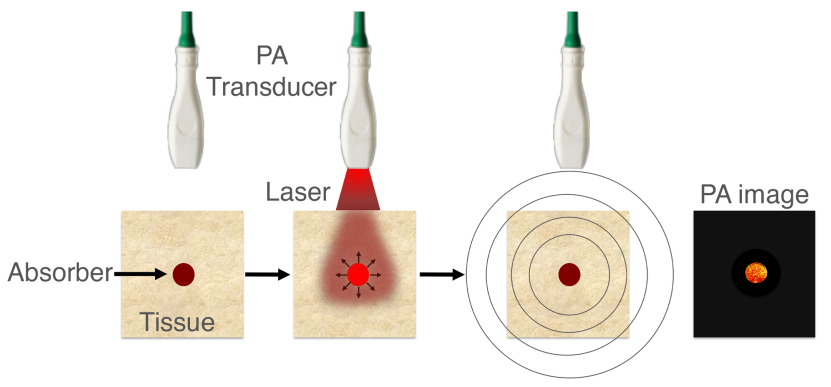

B

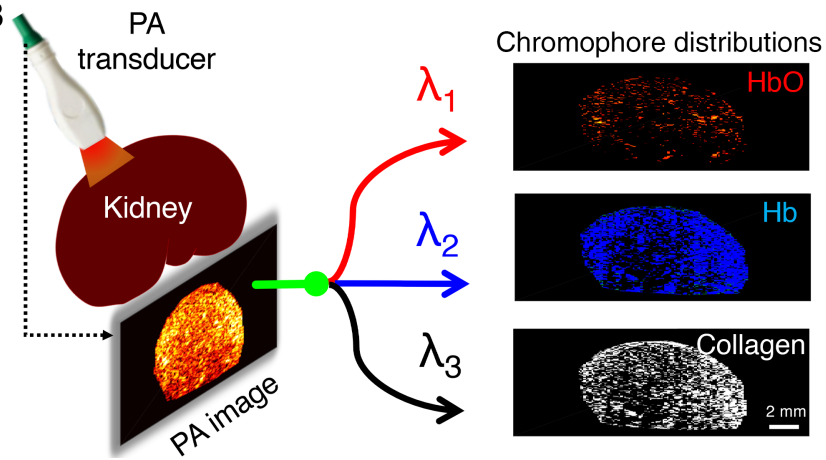

Figure 1. An overview of photoacoustic (PA) imaging with spectral unmixing as a tool to noninvasively quantify renal collagen content. (A) In photoacoustic imaging, a nanosecond (ns) pulsed laser illuminates tissue with light of varying wavelengths. Optical tissue chromophores absorb light at specific wavelengths, leading to thermal expansion that generates an acoustic wave, which is captured by an ultrasound probe. Image reconstruction of these ultrasound waves can provide information on the concentration and location of these chromophores. (B) Using spectral unmixing, PA images acquired at multiple wavelengths $(\lambda)$ are input to the unmixing algorithm, which then can faithfully separate out the individual contributions of oxyhemoglobin (HbO), deoxyhemoglobin $(\mathrm{Hb})$, and collagen. The resulting PA chromophore maps provide the spatial distribution and estimates of the hemoglobin and collagen content within the imaged kidney.

PA imaging can accurately detect murine kidney fibrosis. Having demonstrated the potential of PA imaging as a noninvasive tool to quantify collagen content in simple blood-collagen gels, we next examined whether our PA technique would maintain its imaging accuracy in a more complex and heterogeneous structure, such as the kidney. For these experiments, 2-dimensional (2D) PA imaging of whole mouse kidney (Figure 3A) was performed at various time points following left-sided unilateral ureteral obstruction (UUO). Following obstruction, the left kidney develops progressive and highly reproducible fibrosis over the course of 7-14 days (Figure 3B) (29). PA imaging was performed using a single $2 \mathrm{D}$ longitudinal cross-section through the longest part of each kidney. Compared with mice undergoing sham surgery, 2D PA imaging using light ranging from 680-930 nm demonstrated marked changes in generated PA signals in the fibrotic left kidney (Figure 3C). Total scanning time per kidney was less than 1.5 minutes. Application of our unmixing algorithm using the optimal wavelengths identified in our phantom experiments $(680,725$, and $755 \mathrm{~nm})$ demonstrated that PA detected significant increases in collagen content in the injured left kidney at 7 days after UUO, with further increases noted by 14 days after surgery (Figure 3, B and D). As expected, no increases in collagen content were detected in the healthy right kidney at any time point (Figure 3D). Gold standard histologic quantification of fibrillar collagen using Picrosirius red (PSR) staining at these same time points (Figures 3, B and E) closely mirrored our PA imaging findings. Similar findings were noted when other histologic stains for fibrosis were used (Supplemental Figure 2). Importantly, correlation analysis (Figure 3F) demonstrated striking agreement between our 2D PA imaging-based scores and gold standard histology measurements (Pearson $r=0.89-1.00, P<0.05$ comparing PA-based collagen scores versus either PSR or type I collagen IHC-based collagen measurements). Similarly, our 2D PA-derived mean collagen scores correlated strongly with $\alpha$-smooth muscle actin ( $\alpha$-SMA) staining, a marker of activated fibroblasts (Figure 3F), as well as mRNA levels of various fibrosis-associated genes, including Colla1, the gene encoding type I collagen (Supplemental Figure 3). 

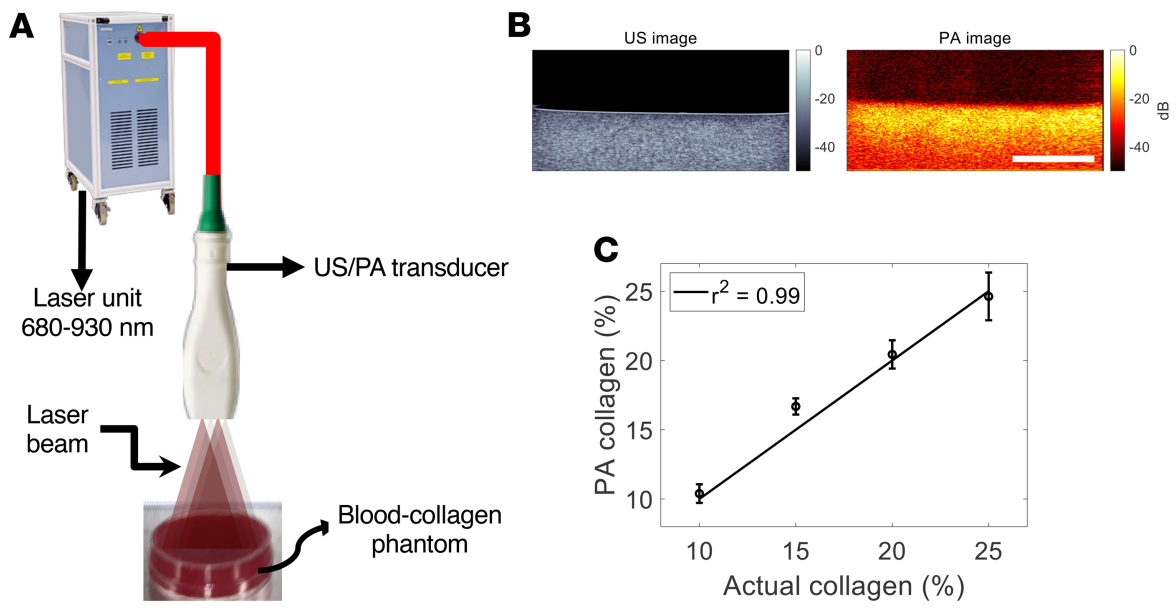

Figure 2. Development and validation of a spectral unmixing algorithm to identify collagen content using photoacoustic (PA) imaging. (A) Photoacoustic (PA) imaging setup for blood-collagen phantom gels. (B) Representative coregistered ultrasound (US) and PA images (at $850 \mathrm{~nm}$ ) of a blood-collagen phantom. Scale bar: $10 \mathrm{~mm}$ (applies to both images). (C) Validation of our spectral unmixing algorithm to quantify collagen. Data represent mean \pm SD, with $n=60$ measurements per phantom $(n=4)$. The $r^{2}$ denotes the goodness of the linear fit, derived from univariate linear regression. $\mathrm{dB}$, decibels.

Three dimensional PA imaging can quantify intrakidney variations in collagen content. Scar deposition within the kidney is characteristically heterogeneous in distribution. This heterogeneity can impair accurate assessment of fibrotic burden when relying only on small tissue samples for histologic analysis (30). Moreover, in areas of severe fibrosis, little renal parenchyma may remain, making histologic identification and analysis of other processes besides fibrosis extremely difficult. Thus, development of a technique that not only assesses global fibrotic burden (as shown using our 2D imaging technique described above), but that also characterizes intrarenal variations in collagen content (to potentially guide the location of a biopsy to assess nonfibrotic processes), would be highly advantageous. To assess whether PA imaging has sufficient resolution to detect spatial heterogeneity in fibrosis content, we performed 3-dimensional (3D) imaging of the entire mouse kidney. 3D PA imaging was performed for all kidneys at intervals of $150 \mu \mathrm{m}$ across the entire length of the organ (Figure 4A). Scanning was completed in less than 5 minutes per kidney. Fibrosis was measured histologically from predefined sections within the kidney (Figure 4A), with the resulting histologic values being directly compared with collagen scores derived from PA images taken at the precise locations of the histologically stained sections. Using this strategy, we observed significant PA-measured fluctuations in collagen content across the kidney that, importantly, was closely mirrored by gold standard histologic quantification acquired in 3 locations within the kidney (left, middle, and right; Figures 4, B and C).

$3 D$ PA imaging is equivalent to $2 D$ PA imaging in quantifying whole kidney fibrotic burden. To test whether our 3D imaging technique provided additional accuracy in quantifying whole kidney fibrotic burden as compared with our single-slice 2D technique, we averaged collagen content from all the component 3D PA images from a given kidney to generate a mean PA-derived whole kidney fibrosis score. These PA fibrosis scores correlated well with histologic scar estimates of mean whole kidney fibrotic burden using several different stains (Figure 4D), but this relationship was not stronger than that derived from our single-slice $2 \mathrm{D}$ imaging technique (Figure $3 \mathrm{~F}$ ). Our results suggest that a single longitudinal $2 \mathrm{D}$ PA image through the mouse kidney is the most rapid way to accurately assess whole kidney fibrotic burden, while 3D PA offers the benefit of enhanced spatial resolution that enables quantification of intrarenal variations in collagen content.

$P A$ is a viable technique for imaging renal collagen in a clinically relevant setting. Our initial studies examined ex vivo mouse kidneys (roughly $1.0 \times 0.5 \times 0.5 \mathrm{~cm}$ ), which are much smaller than human kidneys (roughly $10 \times 5 \times 5 \mathrm{~cm}$ ). PA imaging depends both on adequate light penetration into tissue (to excite a chromophore) and the temperature of the tissue during imaging (to ensure efficient light-induced thermoelastic expansion) (31). As human kidneys are generally located deep within the retroperitoneum, a site that is inaccessible to most current light delivery technologies (32), in vivo PA imaging of human kidneys has 
A Murine kidneys
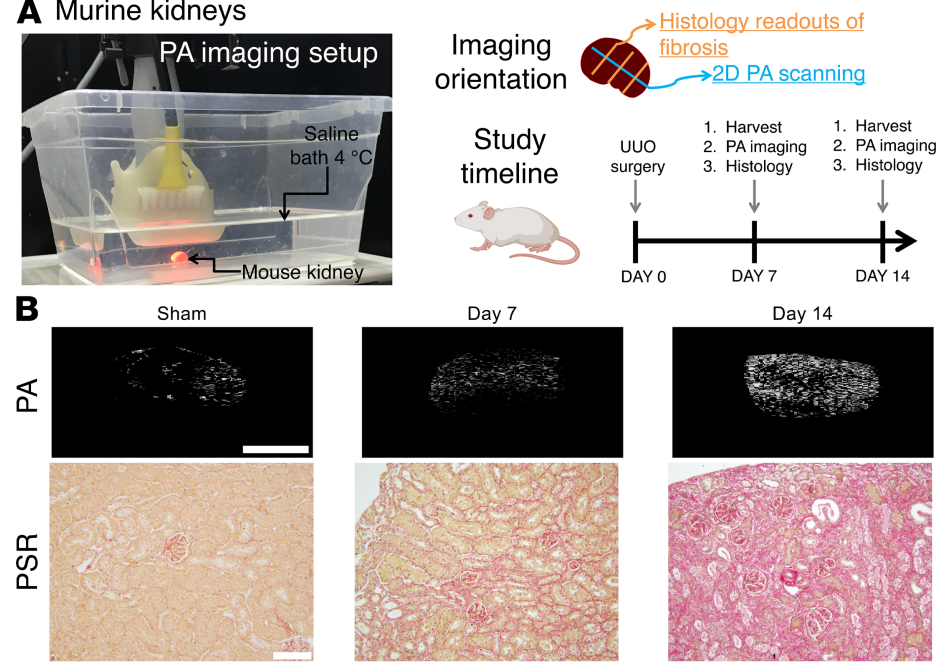

Day 7
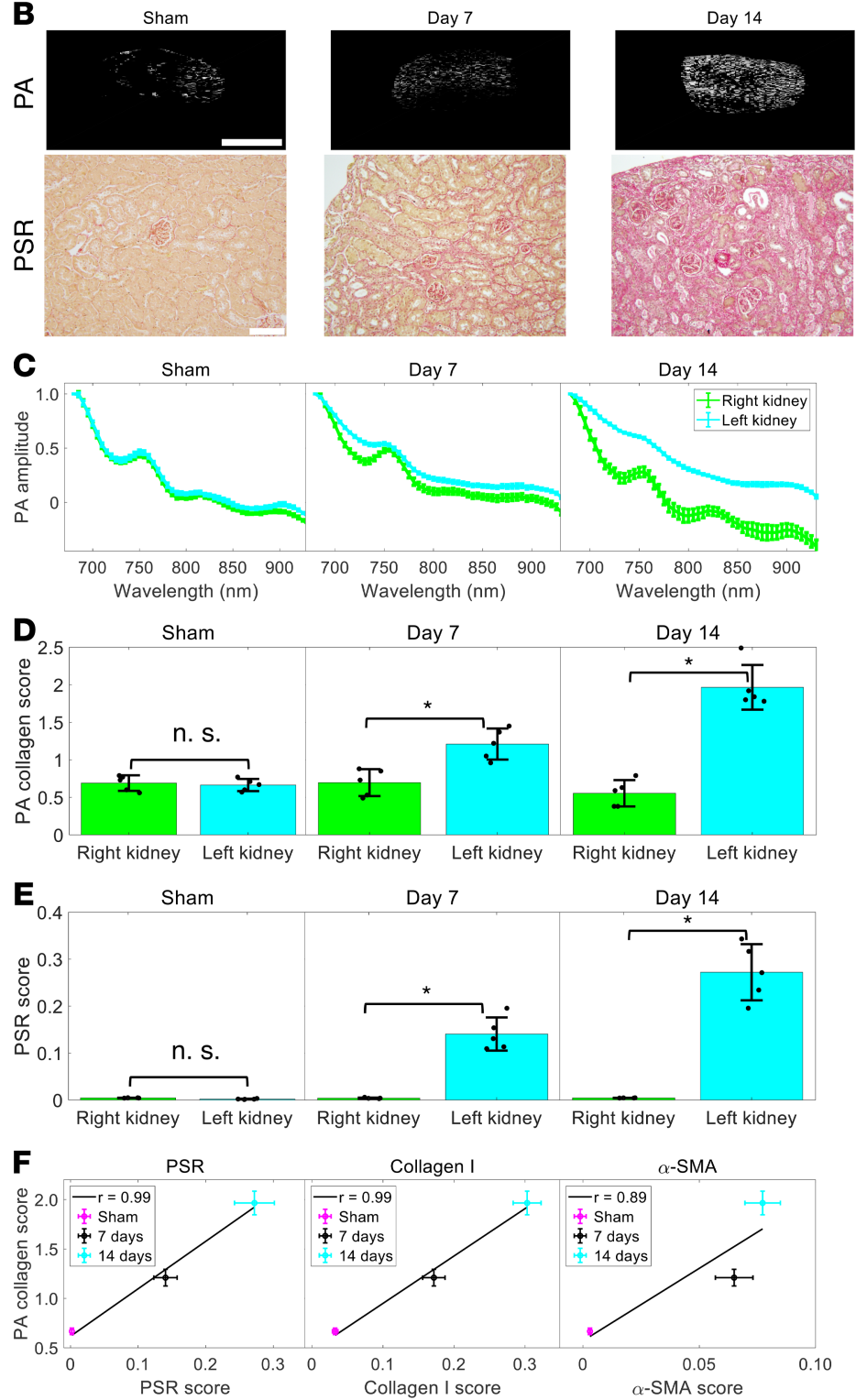

Figure 3. Photoacoustic (PA) imaging detects progressive kidney fibrosis in mice. (A) PA imaging setup and orientations for the UUO-induced mouse kidney fibrosis model, in which the left kidney develops fibrosis. Mouse illustration generated in Biorender (www.biorender.com). (B) Representative PA and Picrosirius red (PSR) histology images at various time points after UUO surgery. Scale bar: $5 \mathrm{~mm}$ (PA images) and $100 \mu \mathrm{m}$ (PSR histology images). (C) Average PA amplitude spectra as a function of optical wavelength. Data represent mean \pm SEM for $n=5$ mice, $n=5$ kidneys $/$ side, and $n=5$ measurements/kidney side. (D) PA-derived collagen scores as a function of time. For all mice undergoing UUO surgery, the right kidney is normal, while the left kidney is fibrotic. Data represent mean $\pm S D$ for $n=5$ mice/kidney side. (E) PSR histology-derived collagen score as a function of time. Data represent mean \pm SEM for $n=5$ mice. For $\mathbf{D}$ and $\mathbf{E}$, a 2-way ANOVA with time as an independent variable was used to assess for time-dependent changes in PA and histologic measures of fibrosis; ${ }^{*} P<0.05$, relative to the right kidney. For $\mathbf{D}$, the $P$ values for the Sham, day 7 , and day 14 comparisons are $P=0.3, P=0.002$, and $P=0.00001$, respectively. For $\mathbf{E}$, the right to left kidney comparison $P$ values are $P=0.1$, $P=0.00001$, and $P=0.000001$ for Sham, day 7 , and day 14 measurements, respectively. (F) Pearson correlation analysis between PA and histology scores for $2 \mathrm{D}$ imaging $(P=0.007$ for PSR, $P=0.007$ for collagen $\mathrm{I}, P=0.03$ for $\alpha$-SMA). 
A
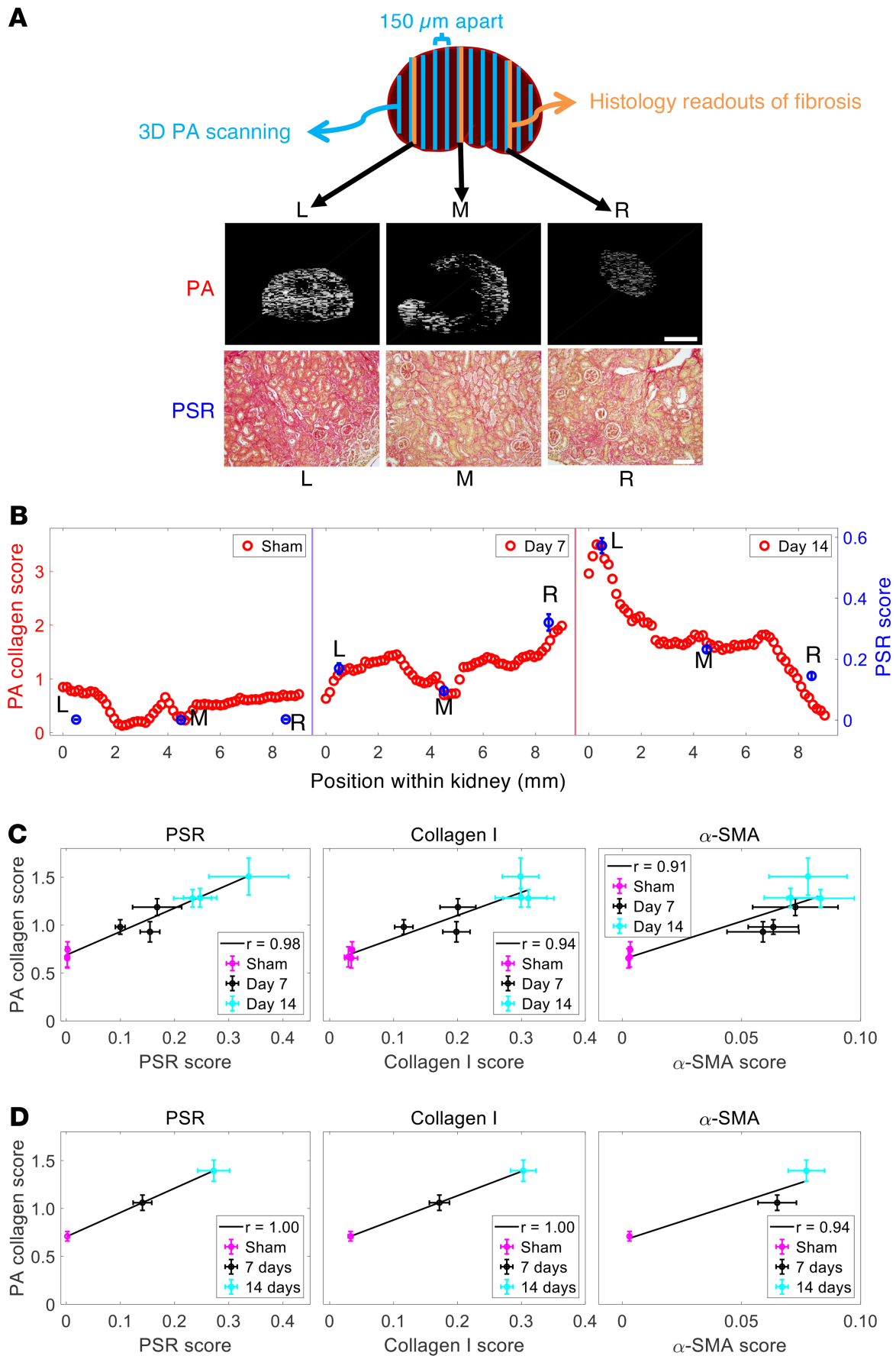

Figure 4. Three-dimensional photoacoustic (PA) imaging accurately measures intra- and interkidney variations in fibrotic burden in a mouse model of fibrosis. (A) PA imaging and histology orientations for mouse kidneys imaged in 3D. Transverse PA image cross-sections were acquired every $150 \mu \mathrm{m}$ (blue lines not to scale), while Picrosirius red (PSR) staining was performed on tissue sections collected at 3 locations (orange lines), covering both poles and the middle of the kidney. Representative PA and PSR histology collagen maps are shown from multiple sections within a fibrotic kidney 14 days after unilateral ureteral obstruction surgery ( $L$, left; $M$, middle; $R$, right). Scale bars: $5 \mathrm{~mm}$ (top) and $100 \mu \mathrm{m}$ (bottom). (B) Intrakidney collagen variation for both PA-derived (left axis) and PSR-derived collagen measurements (right axis). PSR staining measurements were acquired at 3 locations, while PA images were acquired every $150 \mu \mathrm{m}$, across the entire length of the kidney. (C) The mean PSR-based fibrosis scores from each position within the kidney were correlated, with the corresponding mean 3D PA-based collagen scores calculated for each of those same positions ( $P=0.00001$ for PSR, $P=0.0002$ for collagen $\mathrm{I}$, and $P=0.0008$ for $\alpha$-SMA). (D) Pearson correlation analysis between mean 3D PA-based and PSR-based whole kidney fibrosis scores $(P=0.001$ for PSR, $P=0.002$ for collagen I, and $P=0.02$ for $\alpha$-SMA). Data represent mean \pm SEM for $n=5$ mice/time point, with $n$ $=3$ different $\mathrm{PA}$ and histological readings acquired for every mouse, at every time point. 
generally been considered not feasible. During transplantation, however, kidneys are stored ex vivo on ice, often for several hours between their retrieval from the donor and implantation into the recipient. As fibrosis is an important predictor of transplant kidney outcomes that is currently inadequately assessed, we next explored whether PA imaging of the ex vivo transplant kidney might represent an ideal opportunity for a clinically impactful application of our PA technique.

As a first step, we examined whether collagen PA imaging could be performed at $4^{\circ} \mathrm{C}$ on pig kidneys, which are similar in size to human kidneys, to best mimic this clinical setting (Figure 5A). Figure 5B shows the ultrasound and corresponding PA images of pig kidneys imaged at $4^{\circ} \mathrm{C}$ and $37^{\circ} \mathrm{C}$. Given the temperature sensitivity of PA signal generation (33), PA signal intensity was, as expected, reduced at $4^{\circ} \mathrm{C}$. Importantly, however, the amplitude of the detected PA signal remained above noise level for nearly $6 \mathrm{~mm}$ below the kidney surface at both temperatures (Figure 5C). Thus, collagen PA imaging can be successfully performed to roughly $6 \mathrm{~mm}$ below the kidney surface even at $4^{\circ} \mathrm{C}$, the temperature at which human transplant kidneys are stored while awaiting implantation into a recipient (Figure 5, C and D). Importantly, this imaging depth allowed sampling of the majority of the kidney cortex, which — in a healthy kidney — extends to a mean of $8 \mathrm{~mm}$ below the kidney surface (with more damaged kidneys having thinner cortices) (34). Since the PA probe can be scanned across the entire kidney surface, our technique can, thus, image roughly $75 \%$ of the entire renal cortex in a clinically relevant setting that closely mimics human kidney transplantation.

To further assess the translational potential of our PA imaging technique, we next examined whether PA imaging of human kidneys could generate similar results as our proof-of-principle mouse studies. Using a nontransplantable whole human kidney retrieved using the same protocol as that used for actual kidney transplantation, we performed PA imaging at $4^{\circ} \mathrm{C}$ to mimic an ex vivo donor kidney awaiting implantation (Figure 6A). Scanning over the entire kidney surface (Figure 6B) enabled us to image the bulk of the renal cortex. Figure $6, \mathrm{C}$ and D, shows the PA quantification results obtained from imaging both the top and bottom of the whole human kidney, respectively. Much like the mouse kidneys shown in Figure 4B, we noted significant variation in PA-derived collagen scores as we scanned across the human kidney. Remarkably, PA-measured spatial fluctuations in collagen closely mirrored changes noted following histologic quantification of PSR-stained serial slices through the kidney (Figure 6E, Pearson $r=0.97, P<0.05$ ). The ability of PA to quantify collagen heterogeneity within human kidneys was also tested in 5 separate radical nephrectomy specimens (Figure 6F), that - like in the whole human kidney - closely correlated with variations in histologically determined collagen measurements (Figure 6G). Direct comparison of PA collagen scores at various locations within the kidney, with histologic measures of fibrosis derived from gold standard PSR and Masson's Trichrome stained sections at these same locations, demonstrated a very strong correlation across all samples (Figure $6 \mathrm{H}$ ).

To evaluate the performance of PA imaging as a method to quantify overall renal cortical fibrotic burden across multiple human kidney samples, we next compared mean PA-derived collagen scores to gold standard histologic values derived from examination of our human radical nephrectomy specimens. For these analyses, we performed both 2D and 3D PA imaging, as shown in Figure 7A. Figure 7B shows representative collagen maps generated by PA imaging and corresponding images of PSR- and Masson's trichrome-stained tissue sections taken from the same regions within the kidney. As expected, both 2D and 3D PA imaging accurately quantified renal cortical fibrosis (Figure 7, C and D). As we had observed in mouse kidneys, 2D PA collagen imaging was as accurate as 3D PA imaging in assessing renal fibrotic burden, but it was completed in a shorter period of time (2D imaging, 1.5 minutes; 3D imaging, 5 minutes).

\section{Discussion}

Fibrosis is a significant cause of chronic organ injury and dysfunction. In the kidney, fibrosis is a final common pathway of injury triggered by nearly all forms of CKD, and it is a major cause of renal function loss $(12,35)$. Unfortunately, no safe and accurate measure of kidney fibrosis exists. As such, both scientists and clinicians currently must rely on kidney tissue sampling and subsequent measurement of collagen levels via histologic and/or molecular techniques. In rodents, kidneys can only be examined after animal sacrifice. In humans, this process requires an invasive biopsy that not only confers significant bleeding risk, but also samples less than $1 \%$ of the entire kidney. Here, we demonstrate a PA imaging technique that directly measures renal collagen content with remarkable accuracy and without the need for exogenous contrast agents. Unlike any other conventional technology, PA collagen imaging can quickly and noninvasively quantify whole kidney fibrotic burden in mice and fibrotic burden in most of the ex vivo human kidney cortex. 
A

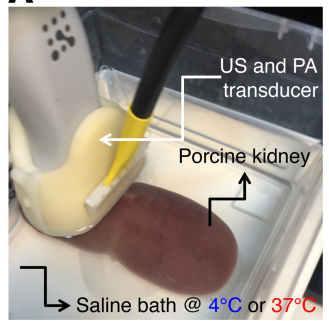

B

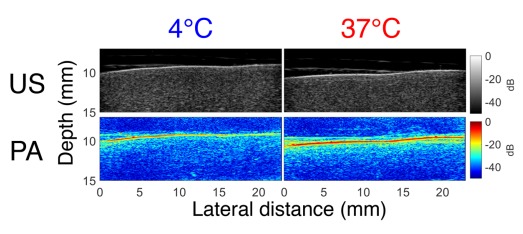

C

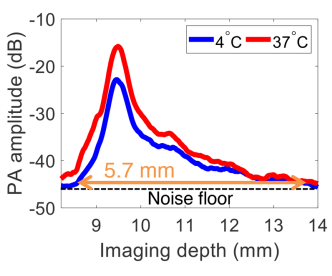

D

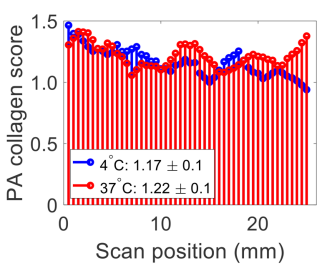

Figure 5. Effect of imaging temperature on PA-based collagen measurements. (A) PA imaging setup for whole porcine kidneys imaged in a $4^{\circ} \mathrm{C}$ or $37^{\circ} \mathrm{C}$ saline bath. (B) Representative US and PA (at $680 \mathrm{~nm}$ ) images at both temperatures. (C) Quantification of the PA imaging depth at both temperatures. The maximal imaging depth was estimated by measuring the distance during which the signal remains above noise level. (D) PA collagen quantification across the kidney as a function of temperature. Scans were performed on 2 separate pig kidneys. A paired, 2-tailed $t$ test with a $95 \% \mathrm{Cl}$ was used to test statistical significance. The average PA collagen scores at $4^{\circ} \mathrm{C}$ and $37^{\circ} \mathrm{C}$ did not statistically differ $(P=0.2)$.

Furthermore, it demonstrates remarkable spatial resolution, being able to accurately capture intrarenal variations in collagen content. Finally, we show that human PA imaging can be performed in a setting that mimics kidney transplantation, a situation in which kidney fibrosis measurements could impact the clinical decision-making process.

Assessment of donor kidney quality is an important step in the transplant process. When first notified of a potential kidney donor, clinicians assess whether a kidney is useable for transplant and next decide which to recipient the kidney should be matched. Given the shortage of available donor kidneys, the ultimate goal of the transplant process is to match each kidney to the most appropriate patient, to maximize the benefit for all recipients. Thus, the least-damaged kidneys ideally should be transplanted into younger recipients who require the longest-lasting kidneys. In contrast, kidneys with some preexisting damage are better matched to older recipients, to avoid longer wait times for less-damaged kidneys that have expected life spans longer than those of the patients themselves $(9,36,37)$.

Clinical tools have been developed to assess donor kidney damage such as fibrosis using surrogate parameters. The most widely used system divides donor kidneys into standard criteria donor (SCD) or expanded criteria donor (ECD) kidneys, based on donor age, renal function, and history of hypertension (38). While easy to use and broadly predictive of outcome, this binary system does not adequately assess donor kidney quality, as many ECD kidneys have better outcomes than some of their SCD counterparts (9). In contrast, kidney biopsy enables detailed histologic assessment, and in particular, biopsy-derived measures of fibrotic burden are powerful predictors of long-term kidney outcomes $(10,11)$. However, biopsy is limited by significant bleeding risk, the potential for sampling bias, the time required for proper staining, and the requirement for 24-hour renal pathology expertise (15). These limitations have rendered biopsy analysis impractical and potentially inaccurate as a means of assessing donor kidney fibrosis. In this context, our demonstration that PA imaging can accurately and noninvasively quantify renal cortical fibrotic burden in a setting that mimics kidney transplantation signals a potential breakthrough for assessment of donor kidney quality.

PA imaging has traditionally focused on generating high-resolution images of superficial capillaries by taking advantage of the strong absorption properties of RBC hemoglobin (39). Recently, attempts have been made to image deeper within the body, with a report demonstrating the ability to image hemoglobin oxygenation within the intestinal wall (40), as well as in breast malignancies (41). Similarly, Lin and colleagues describe a modified light delivery system that allows imaging up to $4 \mathrm{~cm}$ below the skin surface, enabling through-the-skin vascular imaging of the entire human breast (42). Even with these substantial technical advances, the ability to perform in vivo imaging of very deeply situated organs such as the kidney has remained a challenge. To circumvent this issue, we imaged kidneys ex vivo, recognizing that, during transplantation, kidneys are stored outside of the body often for a prolonged period. Mimicking this ex vivo storage state, we were able to image nearly all of the cortex - an important finding, since cortical fibrotic burden is an important determinant of renal outcomes $(10,11)$.

Historically PA imaging studies have focused on the quantification of hemoglobin in tissues, given its strong absorption properties and abundance (32). Recent studies have begun to explore whether collagen, the main component of scar tissue, can also be imaged using PA. To date, these studies have demonstrated that, in small animal preclinical models, PA may be able to image fibrosis in liver (43), 
A

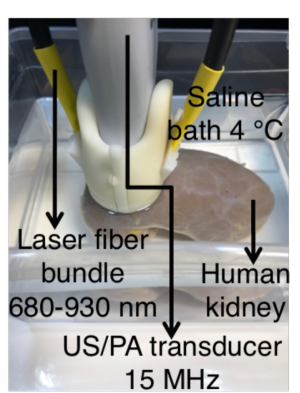

B

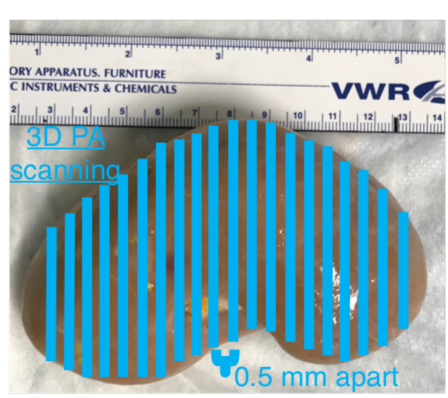

C

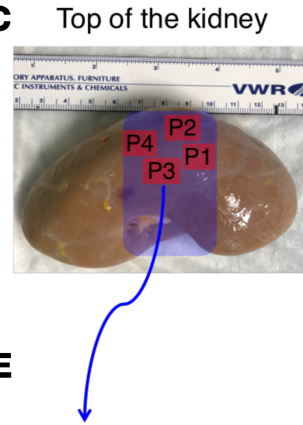

PA collagen map
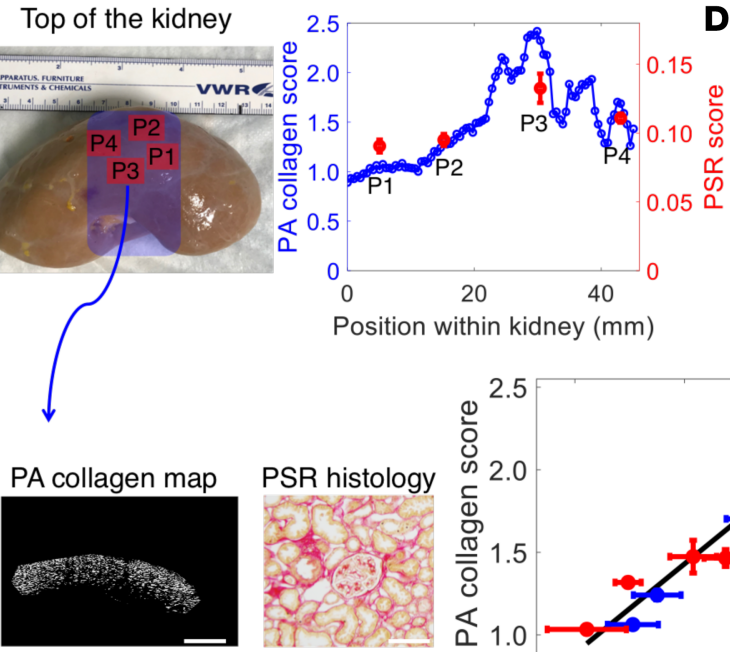

D Bottom of the kidney

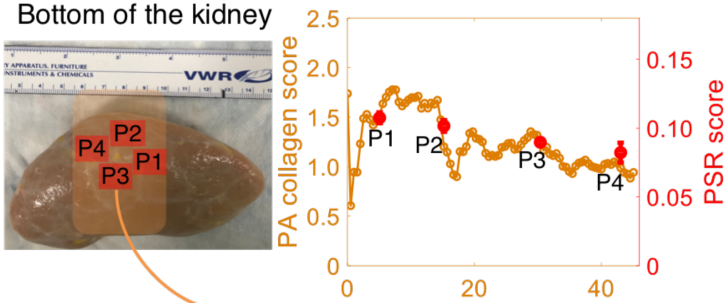

Position within kidney $(\mathrm{mm})$
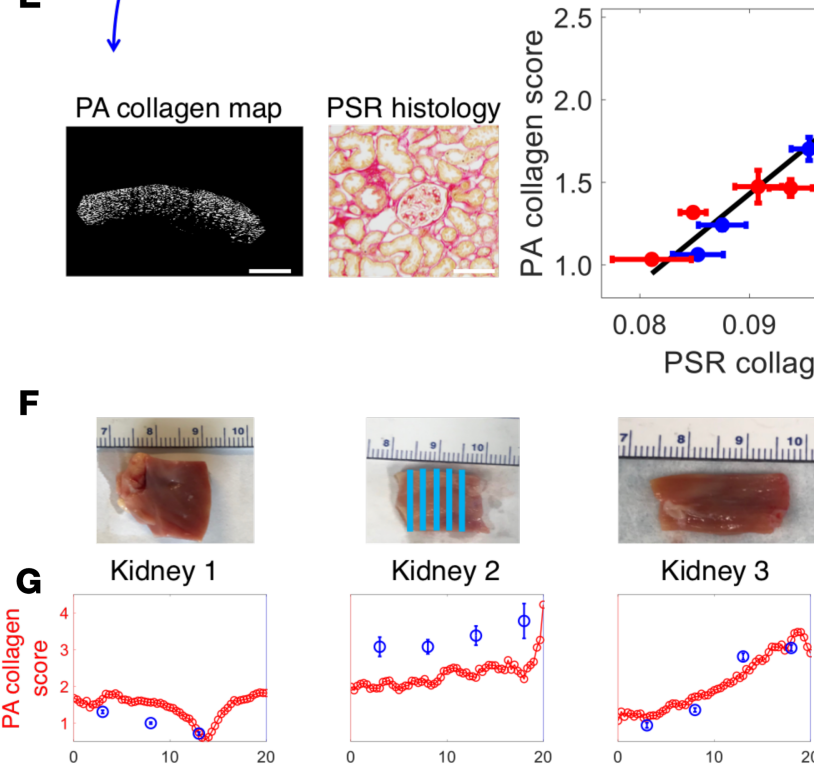

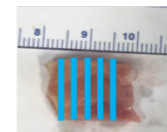

Kidney 2

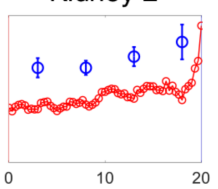

Kidney 3

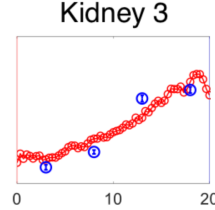

Position within kidney $(\mathrm{mm})$
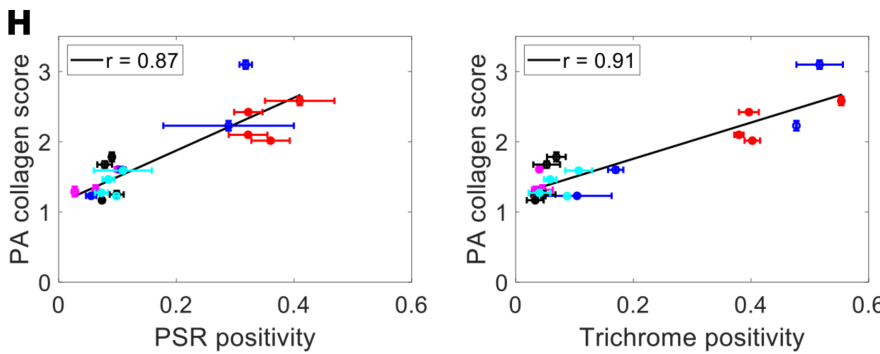

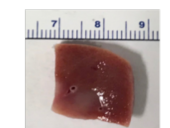

Kidney 4

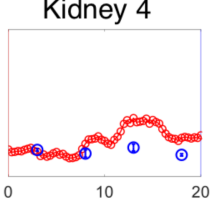

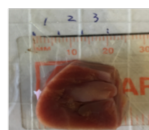

Kidney 5

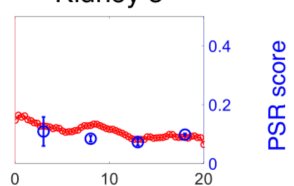

Figure 6. PA imaging can detect intrarenal variations in collagen content in human kidneys. (A) Human kidney PA imaging setup. Imaging was performed in a $4^{\circ} \mathrm{C}$ saline bath. (B) Three-dimensional whole kidney PA imaging strategy. The bottom and top surfaces of the kidney were imaged in the orientation shown, with slices taken every $0.5 \mathrm{~mm}$. (C and D) PA- and Picrosirius red-derived (PSR-derived) collagen scores from selected positions within the top (C) and bottom (D) of the kidney. Labelled positions (P1-P4) represent the locations where histological sampling was performed. (E) Correlation of PA-derived collagen estimates with PSR-derived collagen scores for all measured sites $(n=8)$. The Pearson correlation coefficient was statistically significant $(r=0.97, P=0.0001)$. Representative PA- and PSR-stained images are also shown for both sides of the kidney. Scale bar: $5 \mathrm{~mm}$ (PA) and $100 \mu \mathrm{m}$ (histology). (F) Photographs of the human kidney nephrectomy specimens. The 3D imaging orientation strategy shown in kidney 2 applies to all imaged specimens. (G) Intrakidney collagen variation in nephrectomy specimens, as measured by both PA-derived (left axis, red) and PSR-derived collagen scores (right axis, blue). (H) PA-based collagen scores and histology-derived fibrosis measurements (PSR and Masson's trichrome) were compared at 4 locations within each kidney, using Pearson correlation analysis $\left(P=9 \times 10^{-8}\right.$ for PSR, $P=1 \times 10^{-6}$ for Masson's trichrome). The Data represent mean \pm SEM for $n=4$ PA and histological locations for $n=5$ kidney samples. 


\section{A}
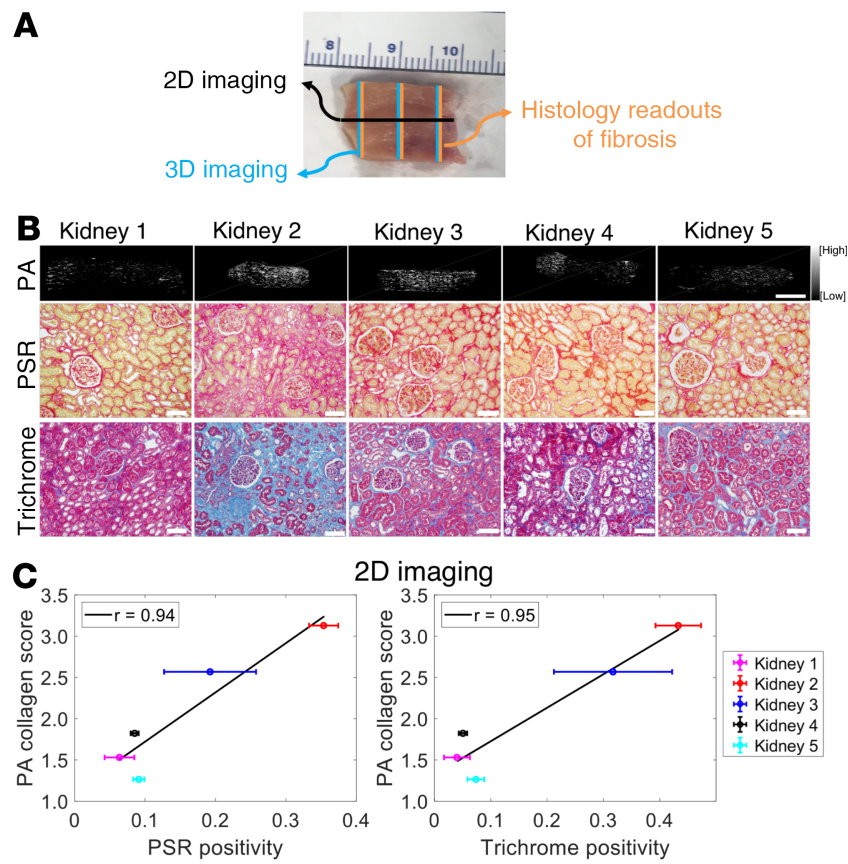

2D imaging
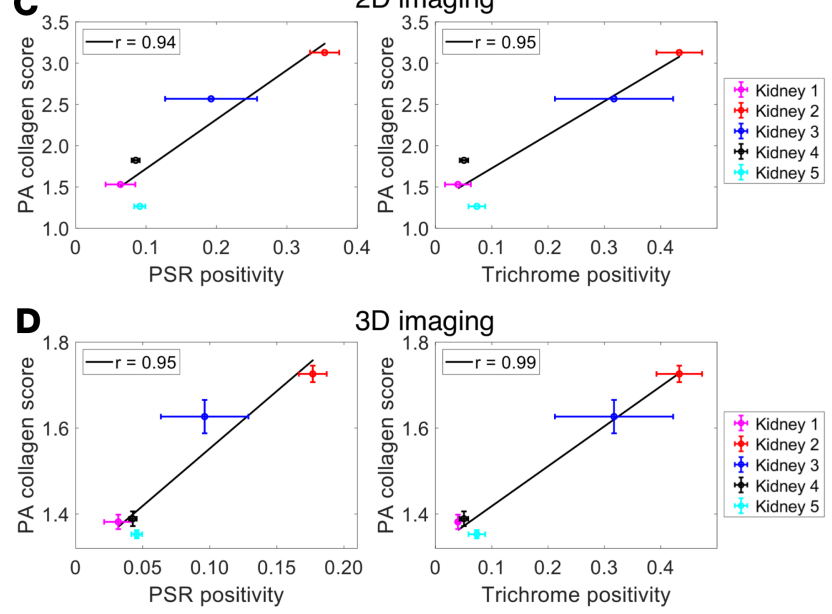

Figure 7. PA imaging can detect interkidney variations in collagen content in human kidneys. (A) PA imaging and histology orientations for human nephrectomy specimens imaged in 2D and 3D. (B) Representative PA and histology collagen maps for 5 kidney samples. Scale bars: $5 \mathrm{~mm}$ (PA) and $100 \mu \mathrm{m}$ (histology). A PA-based collagen score and histology-derived fibrosis measurements for each nephrectomy specimen were calculated. (C and D) Pearson correlation analyses between PA-based collagen scores and histology-derived fibrosis measurements using either 2D ( $P=0.01$ for PSR, $P=0.02$ for Masson's trichrome) (C) or 3D PA imaging ( $P=0.001$ for PSR, $P=0.01$ for Masson's trichrome) (D). Data represent mean \pm SEM for 60 imaging frames per $n=5$ kidneys ( $2 D P A)$ and $n=3$ histology and $n=$ 60 PA locations per sample in the 3D imaging.

intestine $(44,45)$, and skeletal muscle (46). Most of these studies used light with wavelengths greater than $1000 \mathrm{~nm}$, taking advantage of the increased light-absorption properties of collagen in this spectral range to enable stronger collagen-derived PA signal generation (22). Unfortunately, water also efficiently absorbs light at these same wavelengths, limiting the penetration depth and the practical utility of this imaging technique, given the high water content within tissue and the common use of water-based gels as ultrasound transducer coupling agents. Second, these studies imaged fibrosis within large tissue regions, providing an overall measure of fibrotic burden but not demonstrating the ability to accurately quantify intratissue variations in collagen content. In contrast, we developed a PA imaging technique using sub-1000 nm light illumination that accurately estimates collagen concentration with negligible interference from water molecules. This ability to quantify collagen content was maintained over a wide range of hemoglobin levels, suggesting that our spectral unmixing algorithm faithfully distinguishes PA signals produced by collagen from those generated by blood (Figure 1). Finally, we demonstrate that our technique yields sufficiently high resolution to accurately capture intrarenal variations in collagen content, suggesting the potential to image not only differences in collagen amount, but also collagen distribution.

We performed PA imaging of mouse and human kidneys using both 2D and 3D imaging. With 2D PA, we imaged a single longitudinal slice through the kidney. The 2D imaging captured only 1 large section of the kidney but could be performed in less than 1.5 minutes. In contrast, 3D PA imaging captured multiple axial slices, beginning at one pole and progressing to the other pole, such that we were able to image all of 
the mouse kidney and nearly all of the human kidney cortex. This detailed, high-spatial resolution imaging was typically completed in under 5 minutes for mouse kidneys and 15 minutes for a full human kidney. As shown by conventional histology (Figure 4), 3D imaging demonstrated that collagen content is distributed heterogeneously throughout the kidney. Interestingly, however, a single longitudinal 2D slice through the kidney (Figure $3 \mathrm{~F}$ ) gave as an accurate estimate of collagen content as a 3D sweep through the entire kidney (Figure 4, C and D). Thus, in the setting of donor kidney fibrosis assessment during transplantation, when a quick, noninvasive estimate of fibrotic burden is required, a single 1.5-minute transverse $2 \mathrm{D}$ image through the kidney is adequate. For a more comprehensive assessment of collagen distribution, a 5-minute 3D sweep through a mouse kidney or a 15 -minute 3D sweep through a human kidney can also be performed.

This study has some limitations, which form the basis of potential future extensions of this work. First, all of our imaging was performed on ex vivo kidney samples. Ideally, in vivo kidney imaging would be preferred, to enable serial, noninvasive, and nonterminal studies of kidney fibrotic burden. In humans, however, current light-delivery technologies can not reliably illuminate the very deeply situated kidneys, although recent reports suggest that technological advances may soon enable deep-body PA imaging $(32,42)$. In rodents, light delivery is not limiting, but algorithms must be used to correct for the respiratory motion of these small animals $(47,48)$. Development of these respiratory motion correction algorithms for PA collagen imaging could theoretically enable serial, through-the-skin fibrosis measurements of the kidney and potentially other organs in rodents. Such an advance would accelerate preclinical fibrosis research, as the ability to measure fibrosis changes within the same animal could enable true measurements of fibrosis progression and/or regression. Furthermore, the ability to perform serial measurements would also significantly reduce the number of animals required (and associated cost and time) for a given study. Second, we developed our PA algorithm to image type I collagen, which is the most abundant protein found in renal fibrotic matrix $(49,50)$. However, a variety of molecules, such as elastin, tenascin C, and other collagens, are also deposited in scar tissue within the kidney and other organs $(19,51-53)$. The development of PA algorithms to image these proteins could potentially confer even greater diagnostic accuracy for detection of fibrotic tissue.

In summary, we describe a potentially novel PA imaging technique that quickly and noninvasively estimates collagen content in mouse, pig, and human kidneys with striking accuracy. Unlike other conventional or experimental techniques used for fibrosis imaging, PA directly images collagen, a principal constituent of scar tissue, without the need for exogenous contrast agents. This technique visualizes collagen based on its unique light-absorption properties, effectively using collagen as an endogenous contrast agent. We also demonstrate, for the first time to our knowledge, the potential clinical utility of this technique, showing that PA fibrosis imaging can be used to assess human kidney fibrosis in an ex vivo setting that closely mimics cold storage of kidneys at the time of kidney transplantation. Given the importance of kidney fibrosis in determining long-term clinical outcomes, the ability of PA imaging to accurately assess donor kidney fibrotic burden has the potential to influence how kidneys are accepted and allocated.

\section{Methods}

\section{Animal experiments}

Male WT C57BL/6 mice were purchased from Charles River Laboratories. Mice were kept on a 12-hour light/dark cycle with ad libitum access to food and water.

UUO murine kidney fibrosis model. Six- to 8-week-old male WT C57BL/6 mice underwent sham ( $n=$ $5)$ or left-sided UUO surgery $(n=10)$ as previously described (54). Briefly, a left-sided flank incision was made in anesthetized mice, and the left kidney and ureter were identified. For UUO animals, the left ureter was then obstructed with two 4-0 silk suture ties just distal to the renal pelvis. The uninjured right kidney served as a healthy control kidney that did not develop fibrosis. Separate sham-operated animals were also generated as additional controls. In these mice, a similar flank incision was made, but the ureter was not obstructed. UUO mice were divided into 2 groups that varied in their degree of induced fibrosis: (a) mice sacrificed at 7 days after surgery $(n=5)$ and (b) mice sacrificed at 14 days after surgery $(n=5)$. The sham mice were sacrificed at 14 days after sham surgery.

Pig kidney retrieval. Kidneys from 2 Yorkshire pathogen-free female pigs from Lifetime Solutions (pig 1, $46.4 \mathrm{~kg}$; pig 2, $41.7 \mathrm{~kg}$ ) were used for analysis. Pigs were euthanized via an overdose of euthanyl (0.2 $\mathrm{mL} / \mathrm{kg}$; Bimeda-MTC Animal Health Inc., catalog 11940222) and kidneys were retrieved immediately following sacrifice. At the time of sacrifice, $20 \mathrm{~mL}$ of whole kidney pig blood was collected in $\mathrm{K}_{2}$ ETDA 
vacutainers (Thermo Fisher Scientific, catalog 02-689-4) to prevent clotting and for use in the blood-gelatin phantoms. Whole pig kidneys were then subjected to imaging as detailed below. For both pigs, the left kidney was first imaged in a phosphate buffered saline (PBS, MilliporeSigma, catalog 524650) bath kept at $37^{\circ} \mathrm{C}$ (immediately after retrieval), followed by 8 -hour storage at $4^{\circ} \mathrm{C}$. The second imaging session was performed in a $4^{\circ} \mathrm{C}$ PBS bath to examine the impact of temperature on the quantification of collagen at clinically relevant imaging depths.

\section{Collection of radical nephrectomy human kidney specimens}

Ethics approval was secured from the Unity Health Toronto Research Ethics Board (REB 18-193) to collect available residual nephrectomy kidney tissue during radical nephrectomy procedures (complete kidney resections) performed at the St. Michael's Hospital site of Unity Health Toronto. A portion of the renal cortex and medulla was collected from the excised kidney ( $n=5$ kidneys). These specimens were submerged in $4^{\circ} \mathrm{C}$ PBS and were imaged within 4-6 hours after collection.

\section{Collection of whole human kidneys}

A nontransplantable human kidney was procured from the International Institute for the Advancement of Medicine (IIAM), under the same Unity Health Toronto Research Ethics Board-approved (REB 18-193) research protocol described above. The IIAM recovers organs not suitable for transplant when authorization for research usage has been obtained from the donor's family. The kidney was collected and stored on ice during transport according to standard clinical kidney transplantation protocols to minimize ischemic injury, and it was imaged within 24 hours after collection.

\section{Construction of collagen phantoms}

To validate the accuracy of our PA spectral unmixing algorithm, collagen phantoms were constructed. The phantoms consisted of porcine skin gelatin, which is composed mostly of type I collagen (55) (MilliporeSigma, catalog 9000-70-8), admixed with whole pig blood. Varying amounts of gelatin and pig blood were mixed to form different phantoms. The weight-to-volume concentration of the gelatin powder was $10 \%, 15 \%, 20 \%$, and $25 \%$ in relation to the whole blood concentration of $90 \%, 85 \%, 80 \%$, and $75 \%$, respectively. Whole blood was collected from the great saphenous vein of the same pigs used for kidney retrieval. In order to construct each phantom, PBS was heated to $35^{\circ} \mathrm{C}$ before the gelatin powder was slowly added. The suspension was magnetically stirred to achieve homogeneity until it reached $75^{\circ} \mathrm{C}$, and the gelatin was entirely dissolved. The solution was cooled to $37^{\circ} \mathrm{C}$ before adding the appropriate volume of whole pig blood. The blood-collagen suspension was added to $35-\mathrm{mm}$ tissue culture dishes (Corning Inc., catalog 353001 ) and was kept at $4^{\circ} \mathrm{C}$ for 24 hours in order to achieve full solidification before imaging.

\section{PA imaging}

Imaging of collagen phantoms, as well as imaging of mouse, pig, and human kidneys, was performed using the VevoLAZR-X system (Fujifilm VisualSonics Inc.) (56-58). This commercially available system uses an $\mathrm{Nd}$ :YAG laser $512 \mathrm{~nm}$ source through an optical parametric oscillator operating at $10 \mathrm{~Hz}$ pulse repetition frequency. The hand-held linear array probe (15 MHz center frequency; 256 acoustic elements; resolutions, $100 \mu \mathrm{m}$ axial, $200 \mu \mathrm{m}$ lateral, and $1.2 \mathrm{~mm}$ elevational) is attached to a laser capable of emitting $6 \mathrm{~ns}$ short laser pulses $(680-930 \mathrm{~nm})$ from 2 rectangular strips located at a $30^{\circ}$ angle on both sides of the acoustic aperture of the transducer. Coregistered ultrasound and PA images are recorded at a frame rate of $5 \mathrm{~Hz}$. The system also provides full access to the radiofrequency (RF) signals for PA images collected at each wavelength of illumination, permitting the development of custom algorithms for the accurate quantification of collagen in whole kidneys. The probe is mounted to a $3 \mathrm{D}$ motor, which can perform scanning at micron step sizes, permitting high-resolution collagen quantification across maximal volumes of kidney tissue.

For all kidneys and collagen phantoms, a spectral sweep was first performed in the 680-930 nm optical window and repeated a total of 5 times. This initial sweep was followed by 2D imaging, during which 60 PA frames were recorded in the same location of the specimen and at a prespecified cross-section in human nephrectomy specimens (Figure 3A and Figure 7A), at each of the 3 key wavelengths identified as most optimal for unmixing collagen. We collected 60 frames to average the signal and improve the signal/noise ratio. Following this, 3D PA scanning was performed on each specimen, at each of these 3 wavelengths, to quantify the heterogeneity of collagen distribution within each kidney (Figure 4A and Figure 6, B and F). The step 
size employed was $150 \mu \mathrm{m}$ for mouse kidneys and human nephrectomy specimens; 130 sequential frames were collected for the radical nephrectomy samples (total length covered $20 \mathrm{~mm}$ ), and 60 sequential frames were collected for the mouse kidneys (total length covered $9 \mathrm{~mm}$ ). For the whole pig and human kidneys, 3D imaging was performed on both sides of the kidney, with a step size of $0.5 \mathrm{~mm}$, covering $25 \mathrm{~mm}$ in length for pig kidneys and $45 \mathrm{~mm}$ in length for the human kidney during 50 and 90 acquired sequential frames, respectively. These scan step sizes were well below the elevational resolution of the imaging transducer $(1.2 \mathrm{~mm})$, permitting detailed probing of collagen distribution. With the exception of the pig kidneys, all imaging was performed in a $4^{\circ} \mathrm{C}$ saline bath to mimic the temperature at which kidneys are stored before transplantation.

Quantification of collagen concentration through PA spectral unmixing. PA-based quantification of the concentration of any optical absorber relies on differences in the optical absorption of the various chromophores present within a studied tissue (23). Supplemental Figure 1 shows the optical absorption spectra for the 3 dominant chromophores in the kidney: oxyhemoglobin $(\mathrm{HbO})$, deoxyhemoglobin $(\mathrm{Hb})$, and collagen. Based upon differences in these spectra, we developed a custom algorithm, patent pending, for estimating the collagen content within ex vivo kidneys in the presence of varying degrees of blood, and consequently, $\mathrm{HbO}$ and $\mathrm{Hb}$ levels. Full details of the unmixing algorithm are presented in Supplemental Methods.

Briefly, linear spectral unmixing was implemented by fitting the PA spectrum measurements across multiple wavelengths to the linear combination of the dominant chromophores present in the kidney $(\mathrm{HbO}$, $\mathrm{Hb}$, and collagen). Restrictions were implemented to improve chromophore quantification by limiting the algorithm's output between $0 \%$ and $100 \%$ to avoid nonphysical solutions (e.g., oxygen saturation $<0 \%$ ) and requiring that concentrations of all chromophores sum to $100 \%$. Accurate quantification relied on identifying the optimal wavelengths for collagen detection by minimizing the variance inflation factor (VIF) from the extinction coefficients of each chromophore (59). The VIF is a metric that quantifies the stability of a solution by detecting the multicollinearity of an input matrix (in this case, the extinction coefficients). VIF minimization was implemented in our studies, and 680, 725, and $755 \mathrm{~nm}$ were identified as the optimal wavelengths for estimating collagen concentration in the presence of $\mathrm{HbO}$ and $\mathrm{Hb}$. A unitless $\mathrm{PA}$ collagen score (corresponding to its concentration) was computed as the average of each image acquired during $2 \mathrm{D}$ and 3D imaging. For more details on PA spectral unmixing, see the Supplemental Methods.

Tissue collection, preparation, and histochemistry

Following PA imaging, each kidney (mouse, pig, and human) was immersed in 10\% neutral buffered formalin for immediate fixation and/or stored in RNAlater (Invitrogen, catalog 7020). Formalin-fixed tissues were embedded in paraffin and sectioned before staining with PSR (MilliporeSigma, catalog 365548), Masson's trichrome (MilliporeSigma, catalog HT15-1KT), or antibodies against $\alpha$-SMA (1:100 dilution, Agilent Dako, catalog M0851) and type I collagen (collagen I, Southern Biotechnology, catalog 1310-01). Random, nonoverlapping $20 \times$ cortical images were taken by a blinded observer using an upright Olympus light microscope and then analyzed in blinded fashion using Aperio Imagescope software as previously described (54). The collagen content within each image was defined as the percentage of positive pixels. Histology-derived collagen scores for any given tissue section were then computed by averaging the individual scores from 4-6 nonoverlapping cortical images.

\section{Semiquantitative reverse transcription PCR}

Tissue stored in RNAlater was homogenized and RNA isolated with TRIzol (Invitrogen, catalog 15596108). RNA was then reverse transcribed into cDNA with Multiscribe Reverse Transcriptase (Thermo Fisher Scientific, catalog 4311235), and levels of Col1a1, Col3a1, Col4a1, Acta2, and/or Gapdh were quantified. Primer sequences are summarized in Supplemental Table 1. Experiments were performed in triplicate. Data analyses were performed using the Applied Biosystems Comparative Ct method. All values were referenced to the mRNA transcript levels of the housekeeper gene Gapdh.

\section{Evaluating the accuracy of inter-kidney PA estimates of collagen concentration}

The average PA collagen score was computed for both the $2 \mathrm{D}$ and $3 \mathrm{D}$ imaging orientations in the mouse and human kidneys. For 2D imaging, the mean collagen score for each kidney was calculated by averaging the collagen scores derived from the individual 60 temporal PA images acquired at each wavelength, at the same spatial location within the kidney. For 3D imaging, a mean PA-derived collagen score for each mouse kidney or human radical nephrectomy specimen was calculated by averaging the collagen score for each 
image captured as the probe was moved sequentially across the kidney. For comparisons of the PA imaging scores for mouse kidneys and nephrectomy samples with histology (PSR, type I collagen, and $\alpha$-SMA), Pearson linear correlation analysis was used (60). The histologically derived fibrosis scores from the 3-4 locations within the kidney were averaged to produce a mean histology fibrosis score for each group of animals (sham, day 7, or day 14) or nephrectomy samples (kidneys $1-5$ ). This was correlated with the 2D (Figures $3 \mathrm{~F}$ and Figure 7C) and 3D (Figures 4D and Figure 7D) PA fibrosis scores. For mouse kidneys, the mean PA-derived kidney score was also correlated against fibrosis-associated transcript levels (Col1a1, Co13a1, Co14a1, Acta2).

\section{Evaluating the accuracy of intrakidney PA estimates of collagen concentration}

For mouse kidneys and human radical nephrectomy specimens, intrakidney collagen variation was assessed by comparing the 3D PA-derived collagen scores collected at specific locations within the kidney against the average PSR- and Masson's trichrome-derived fibrosis scores derived from tissue sections collected at those same locations. These histological sections were acquired at 3-4 locations within the sample, taken approximately $3 \mathrm{~mm}$ apart for the mouse kidneys (Figure 4A) and $5 \mathrm{~mm}$ apart for the human kidney tissue (Figure 7A). Correlations with imaging were performed by averaging the 3D PA score calculated from 10 imaging frames spanning $1.5 \mathrm{~mm}$ in the location where the histological sample was retrieved.

For the whole human kidney, the PSR-derived collagen scores from the top and bottom of the kidney at 4 locations per side were compared with the corresponding PA collagen scores acquired from 3D scanning at the same locations using Pearson linear correlation coefficient analysis (Figure 6, C and D). As described above, PA collagen scores for a given location were calculated from 10 imaging frames spanning $5 \mathrm{~mm}$ in each location. For the radical nephrectomy kidney specimens, the ability of PA imaging to capture intrakidney collagen variation was assessed by comparing the average PSR and Masson's trichrome histology scores acquired at 3-4 locations within the sample, with 3D PA-derived collagen scores calculated from 10 imaging frames spanning $1.5 \mathrm{~mm}$ in each location. The corresponding $P$ value was computed for each correlation, and the correlation coefficient was considered statistically significant for $P<0.05$ (Matlab 2018b, MathWorks Inc.)

\section{Statistics}

As described above, Pearson linear correlation coefficient analysis was used to assess the degree of correlation between our PA estimates of renal collagen content and gold standard measures of fibrosis. Two-way ANOVA with time as an independent variable was used to assess for time-dependent changes in PA and histologic measures of fibrosis. A paired, 2-tailed $t$ test with a 95\% CI was used to test statistical significance. A variable with a $P$ value of less than 0.05 was considered statistically significant, and post hoc comparisons using Tukey's least significant differences identified 95\% CI to determine pairs that were significantly different (61). Statistical analysis was performed using Matlab 2018b (MathWorks Inc.).

\section{Study approval}

All animal studies were approved by the St. Michael's Hospital Animal Ethics Committee and conformed to the Canadian Council on Animal Care guidelines. As mentioned above, ethics approval was secured from the Unity Health Toronto Research Ethics Board (REB 18-193) to collect available residual nephrectomy kidney tissue during radical nephrectomy procedures (complete kidney resections) performed at the St. Michael's Hospital site of Unity Health Toronto. Similarly, ethics approval to procure a nontransplantable human kidney from the IIAM was also secured under the same Unity Health Toronto Research Ethics Board-approved (REB 18-193) research protocol.

\section{Author contributions}

Conceptualization was contributed by $\mathrm{EH}, \mathrm{MCK}$, and DAY; methodology was contributed by $\mathrm{EH}, \mathrm{XH}$, MCK, and DAY; software was contributed by EH and MNF; validation, formal analysis, investigation, and data curation were contributed by $\mathrm{EH}, \mathrm{XH}$, and TZ; provision of study materials, reagents, materials, patients, laboratory samples, animals, instrumentation, computing resources, or other analysis tools were contributed by AK, MO, MF, KTP, VM, WLL, MCK, and DAY; writing and preparation of the original draft were contributed by $\mathrm{EH}$ and DAY; writing, review, and editing were contributed by $\mathrm{EH}, \mathrm{XH}, \mathrm{MNF}$, AK, MO, KTP, WLL, MCK, and DAY; visualization was contributed by EH, MCK, and DAY; and supervision, project administration, and funding acquisition were contributed by $\mathrm{EH}, \mathrm{MCK}$, and DAY. 


\section{Acknowledgments}

This work was supported by a Collaborative Health Research Project grant cofunded by Canadian Institutes of Health Research (CIHR) and the Natural Sciences and Engineering Research Council of Canada (NSERC; awarded to MCK and DAY), a Banting and Best Diabetes Centre Pilot and Feasibility grant (awarded to MCK and DAY), a Canadian Society of Transplantation and Canadian National Transplant Research Program Basic Research Catalyst grant (awarded to MCK and DAY), and funds from the St. Michael's Hospital Foundation (awarded to DAY). EH is supported by a Vanier Canada Graduate Scholarship. WLL is a Canada Research Chair in Mechanisms of Endothelial Permeability, and work in his laboratory is funded by a Collaborative Health Research Project from CIHR and NSERC. DAY is supported by a CIHR New Investigator Award. The authors wish to thank research staff Lindita Rapi, Weiqiu Yuan, and Michelle Nash for assistance in ethics board approvals, data collection, and study coordination. Danielle Bince's assistance with handling pig kidneys is also gratefully acknowledged.

Address correspondence to: Darren A. Yuen, Room 509, 5th Floor, Li Ka Shing Knowledge Institute, St. Michael's Hospital, 209 Victoria Street, Toronto, Ontario Canada M5B 1T8. Phone: 416.864 .6060 ext 77336; Email: darren.yuen@utoronto.ca.

1. Canadian Institute for Health Information. Canadian Organ Replacement Register Metadata (CORR). Canadian Institute for Health Information. https://www.cihi.ca/en/canadian-organ-replacement-register-metadata-corr. Accessed April $28,2020$.

2. USRDS Coordinating Center. 2019 Annual Data Report. United States Renal Data System (USRDS). https://www.usrds.org/ adr.aspx. Accessed April 28, 2020.

3. Wolfe RA, et al. Comparison of mortality in all patients on dialysis, patients on dialysis awaiting transplantation, and recipients of a first cadaveric transplant. N Engl J Med. 1999;341(23):1725-1730.

4. Port FK, Wolfe RA, Mauger EA, Berling DP, Jiang K. Comparison of survival probabilities for dialysis patients vs cadaveric renal transplant recipients. JAMA. 1993;270(11):1339-1343.

5. Laupacis A, et al. A study of the quality of life and cost-utility of renal transplantation. Kidney Int. 1996;50(1):235-242.

6. The Kidney Foundation of Canada. Facing the Facts - The Kidney Foundation of Canada | La Fondation canadienne du rein. https://kidney.ca/KFOC/media/images/PDFs/Facing-the-Facts-2020.pdf

7. Schold J, Srinivas TR, Sehgal AR, Meier-Kriesche HU. Half of kidney transplant candidates who are older than 60 years now placed on the waiting list will die before receiving a deceased-donor transplant. Clin J Am Soc Nephrol. 2009;4(7):1239-1245

8. Port FK, et al. Donor characteristics associated with reduced graft survival: an approach to expanding the pool of kidney donors. Transplantation. 2002;74(9):1281-1286.

9. Rao PS, et al. A comprehensive risk quantification score for deceased donor kidneys: the kidney donor risk index. Transplantation. 2009;88(2):231-236.

10. De Vusser K, et al. The predictive value of kidney allograft baseline biopsies for long-term graft survival. J Am Soc Nephrol. 2013;24(11):1913-1923.

11. Srivastava A, et al. The Prognostic Value of Histopathologic Lesions in Native Kidney Biopsy Specimens: Results from the Boston Kidney Biopsy Cohort Study. J Am Soc Nephrol. 2018;29(8):2213-2224.

12. Steegh FM, et al. Early loss of peritubular capillaries after kidney transplantation. J Am Soc Nephrol. 2011;22(6):1024-1029.

13. Naesens $\mathrm{M}$, et al. Chronic histological damage in early indication biopsies is an independent risk factor for late renal allograft failure. Am J Transplant. 2013;13(1):86-99.

14. Tampe D, Zeisberg M. Potential approaches to reverse or repair renal fibrosis. Nat Rev Nephrol. 2014;10(4):226-237.

15. Wang CJ, Wetmore JB, Crary GS, Kasiske BL. The Donor Kidney Biopsy and Its Implications in Predicting Graft Outcomes: A Systematic Review. Am J Transplant. 2015;15(7):1903-1914.

16. Wang HJ, Kjellstrand CM, Cockfield SM, Solez K. On the influence of sample size on the prognostic accuracy and reproducibility of renal transplant biopsy. Nephrol Dial Transplant. 1998;13(1):165-172.

17. Williams WW, Taheri D, Tolkoff-Rubin N, Colvin RB. Clinical role of the renal transplant biopsy. Nat Rev Nephrol. 2012;8(2):110-121.

18. Leung G, et al. Could MRI Be Used To Image Kidney Fibrosis? A Review of Recent Advances and Remaining Barriers. Clin J Am Soc Nephrol. 2017;12(6):1019-1028.

19. Sun Q, et al. Elastin imaging enables noninvasive staging and treatment monitoring of kidney fibrosis. Sci Transl Med. 2019;11(486):eaat4865.

20. Wang LV, Hu S. Photoacoustic tomography: in vivo imaging from organelles to organs. Science. 2012;335(6075):1458-1462.

21. Wang LV, Yao J. A practical guide to photoacoustic tomography in the life sciences. Nat Methods. 2016;13(8):627-638.

22. Yao J, Wang LV. Photoacoustic tomography: fundamentals, advances and prospects. Contrast Media Mol Imaging. 2011;6(5):332-345.

23. Paproski RJ, Heinmiller A, Wachowicz K, Zemp RJ. Multi-wavelength photoacoustic imaging of inducible tyrosinase reporter gene expression in xenograft tumors. Sci Rep. 2014;4:5329.

24. Cox B, Laufer JG, Arridge SR, Beard PC. Quantitative spectroscopic photoacoustic imaging: a review. J Biomed Opt. 2012;17(6):061202.

25. Laufer J, et al. In vivo photoacoustic imaging of mouse embryos. J Biomed Opt. 2012;17(6):061220.

26. Razansky D, et al. Multispectral opto-acoustic tomography of deep-seated fluorescent proteins in vivo. Nat Photonics. 2009;3(7):412-417. 
27. Eddy AA. Molecular insights into renal interstitial fibrosis. J Am Soc Nephrol. 1996;7(12):2495-2508.

28. Ntziachristos V, Ripoll J, Wang LV, Weissleder R. Looking and listening to light: the evolution of whole-body photonic imaging. Nat Biotechnol. 2005;23(3):313-320.

29. Chevalier RL, Forbes MS, Thornhill BA. Ureteral obstruction as a model of renal interstitial fibrosis and obstructive nephropathy. Kidney Int. 2009;75(11):1145-1152.

30. Kirpalani A, et al. Magnetic Resonance Elastography to Assess Fibrosis in Kidney Allografts. Clin J Am Soc Nephrol. 2017;12(10):1671-1679.

31. Petrova IY, et al. Optoacoustic monitoring of blood hemoglobin concentration: a pilot clinical study. Opt Lett. 2005;30(13):1677-1679.

32. Vu T, Razansky D, Yao J. Listening to tissues with new light: recent technological advances in photoacoustic imaging. J Opt. 2019;21(10).

33. Shao P, Cox B, Zemp RJ. Estimating optical absorption, scattering, and Grueneisen distributions with multiple-illumination photoacoustic tomography. Appl Opt. 2011;50(19):3145-3154.

34. Moghazi S, et al. Correlation of renal histopathology with sonographic findings. Kidney Int. 2005;67(4):1515-1520.

35. Cosio FG, et al. Kidney allograft fibrosis and atrophy early after living donor transplantation. Am J Transplant. 2005;5(5):1130-1136.

36. Watson CJ, Johnson RJ, Birch R, Collett D, Bradley JA. A simplified donor risk index for predicting outcome after deceased donor kidney transplantation. Transplantation. 2012;93(3):314-318.

37. Debout A, et al. Each additional hour of cold ischemia time significantly increases the risk of graft failure and mortality following renal transplantation. Kidney Int. 2015;87(2):343-349.

38. Rosengard BR, et al. Report of the Crystal City meeting to maximize the use of organs recovered from the cadaver donor. $A m J$ Transplant. 2002;2(8):701-711.

39. Li L, et al. Single-impulse Panoramic Photoacoustic Computed Tomography of Small-animal Whole-body Dynamics at High Spatiotemporal Resolution. Nat Biomed Eng. 2017;1(5):0071.

40. Knieling F, et al. Multispectral Optoacoustic Tomography for Assessment of Crohn's Disease Activity. N Engl J Med. 2017;376(13):1292-1294.

41. Neuschler EI, et al. A Pivotal Study of Optoacoustic Imaging to Diagnose Benign and Malignant Breast Masses: A New Evaluation Tool for Radiologists. Radiology. 2018;287(2):398-412.

42. Lin L, et al. Single-breath-hold photoacoustic computed tomography of the breast. Nat Commun. 2018;9(1):2352.

43. van den Berg PJ, Bansal R, Daoudi K, Steenbergen W, Prakash J. Preclinical detection of liver fibrosis using dual-modality photoacoustic/ultrasound system. Biomed Opt Express. 2016;7(12):5081-5091.

44. Zhu Y, et al. Identifying intestinal fibrosis and inflammation by spectroscopic photoacoustic imaging: an animal study in vivo. Biomed Opt Express. 2018;9(4):1590-1600.

45. Lei $\mathrm{H}$, et al. Characterizing intestinal strictures of Crohn's disease in vivo by endoscopic photoacoustic imaging. Biomed Opt Express. 2019;10(5):2542-2555.

46. Regensburger AP, et al. Detection of collagens by multispectral optoacoustic tomography as an imaging biomarker for Duchenne muscular dystrophy. Nat Med. 2019;25(12):1905-1915.

47. Schwarz M, Garzorz-Stark N, Eyerich K, Aguirre J, Ntziachristos V. Motion correction in optoacoustic mesoscopy. Sci Rep. 2017;7(1):10386.

48. Aguirre J et al. Precision assessment of label-free psoriasis biomarkers with ultra-broadband optoacoustic mesoscopy. Nat Biomed Eng. 2017;1:0068.

49. Hewitson TD, Boon WC, Simpson ER, Smith ER, Samuel CS. Estrogens do not protect, but androgens exacerbate, collagen accumulation in the female mouse kidney after ureteric obstruction. Life Sci. 2016;158:130-136.

50. Hijmans RS, et al. Urinary collagen degradation products as early markers of progressive renal fibrosis. J Transl Med. 2017;15(1):63

51. Fu H, et al. Tenascin-C Is a Major Component of the Fibrogenic Niche in Kidney Fibrosis. J Am Soc Nephrol. 2017;28(3):785-801.

52. Chiusa M, et al. The Extracellular Matrix Receptor Discoidin Domain Receptor 1 Regulates Collagen Transcription by Translocating to the Nucleus. J Am Soc Nephrol. 2019;30(9):1605-1624.

53. Wang F, et al. Assessment of renal fibrosis in murine diabetic nephropathy using quantitative magnetization transfer MRI. Magn Reson Med. 2018;80(6):2655-2669.

54. Szeto SG, et al. YAP/TAZ Are Mechanoregulators of TGF-B-Smad Signaling and Renal Fibrogenesis. J Am Soc Nephrol. 2016;27(10):3117-3128.

55. Yang H, Shu Z. The extraction of collagen protein from pigskin. J Chem Pharm Res. 2014:6(2):683-687.

56. Needles A, et al. Development and initial application of a fully integrated photoacoustic micro-ultrasound system. IEEE Trans Ultrason Ferroelectr Freq Control. 2013;60(5):888-897.

57. Bok TH, Hysi E, Kolios MC. Simultaneous assessment of red blood cell aggregation and oxygen saturation under pulsatile flow using high-frequency photoacoustics. Biomed Opt Express. 2016;7(7):2769-2780.

58. Hysi E, Wirtzfeld LA, May JP, Undzys E, Li SD, Kolios MC. Photoacoustic signal characterization of cancer treatment response: Correlation with changes in tumor oxygenation. Photoacoustics. 2017;5:25-35

59. Belsley DA, Kuh E, Welsch RE. Detecting and Assessing Collinearity. In: Regression Diagnostics: Identifying Influential Data and Sources of Collinearity. Hoboken, NJ: John Wiley \& Sons, Ltd; 2005:85-191.

60. Fisher RA. Statistical Methods for Research Workers. In: Kotz S, Johnson NL, eds. Breakthroughs in Statistics: Methodology and Distribution. New York, NY: Springer New York; 1992:66-70.

61. Seber GAF, Lee AJ. Linear Regression Analysis. Hoboken, NJ; John Wiley \& Sons; 2012 